\title{
IMPROVING THE QUALITY OF SELF-ORGANIZING MAP BY “DIFFERENT ELEMENTS" COMPETITIVE STRATEGY
}

\author{
LE ANH TU \\ Thai Nguyen University of Information and Communication Technology; \\ anhtucntt@gmail.com
}

\begin{abstract}
cheske
Abstract. A Self-Organizing Map (SOM) has good quality when both of its measures, quantization error (QE) and topographic error (TE), are small. Many researchers have tried to reduce these measures by improving SOM's learning algorithm, however, most results only decrease either QE or TE. In this paper, a method to improve the quality of the map obtained when the SOM's learning algorithm ended is proposed. The proposed method re-adjusts weight vector of each neuron according to cluster's center that neuron represents and optimizes clusters by "different elements" competitive strategy. In this method, QE always decreases each time the competition "different elements" occurs between all neurons, TE may reduce when the competition "different elements" occurs between adjacent neighbors. The experiments are performed on assumed datasets and real datasets. As the results, the average reduction ratio of $\mathrm{QE}$ is from $50 \%$ to $60 \%$, TE gets the average reduction ratio from $10 \%$ to $20 \%$. This reduction ratio is larger than some other solutions but does not need to adjust the parameters for each specific dataset.
\end{abstract}

Keywords. self-organizing map, competitive learning, different elements, quantization error, topographic error.

\section{INTRODUCTION}

The SOM neural network was proposed by Teuvo Kohonen in 1980s [16]. This is a feedforward neural network model, using an unsupervised competitive learning algorithm. The SOM allows mapping data from multi-dimensional space to less dimensional one (normally 2 dimensions), which makes up the feature map of the data. So far, there have been many different variations of SOM proposed [5] and there are many studies showing that feature map's quality of SOM depends greatly on the initialization parameters such as: Kohonen layer size, numbers of training and neighboring radius $[7,10,16,19,29]$.

The quality of SOM's feature map is evaluated based on two criteria, learning quality and projection quality primarily $[3,13,23,27]$. In particular, the learning quality is determined by measuring the QE (demonstrates the data representation accuracy) $[4,16]$ and the projection quality determined by measuring the TE (demonstrates the topology preservation) [2, 15, 20].

In fact, the method of "trying error" is used to choose suitable parameters [16]. According to Chattopadhyay [6], with a particular dataset, the network size is chosen by "trying error" until small QE and TE achieved. Polzlbauer indicates technical correlation between QE and TE [24], TE usually increases when the QE decreases; besides, in case Kohonen layer's size larges, QE reduces but TE rises (i.e. increasing Kohonen layer's size can lead to the map's topographic deformation) and whereas when Kohonen layer's size is too small, TE may be not trustful. The use of small neighboring

(C) 2015 Vietnam Academy of Science \& Technology 
radius also leads to reduce the $\mathrm{QE}$, if neighboring size is smallest, $\mathrm{QE}$ will achieve the minimum value [26].

Beside the "trying error" method to determine an appropriate network configuration, the researches for improving the learning algorithm are also developed by other researchers. Germen $[8,9]$ optimized QE by integrating parameter "hit" when updating the weights of the neurons. The term "hit" means the numbers of excitation of a neuron. As a consequence, the neurons representing major samples will be adjusted less than the neurons representing the minor samples (to ensure no loss of information). Neme [21,22] proposed model SOM with selective refractoriness allows optimizing TE. In this model, the neighboring radius of BMU does not reduce gradually during the learning process, each training time, each neuron in the neighboring radius of BMU will decide whether the next training is influenced by the BMU again or not. Kamimura [14] integrated "firing" rate into distance function in order to maximize input information. The "firing" rate represents the importance of each feature compared to the remaining features. His method reduces both QE and TE, however, its limitation is each dataset needs to do "trying error" to achieve the appropriate "firing". Another research, Lopez-Rubio [18] gave out the cause of the TE due to the self-intersections (Fig.3) as in following definition: A map is self-intersected if and only if there two triples of adjacent units $\{i, j, k\}$ and $\{r, s, t\}$ that satisfy two conditions: $\{i, j, k\} \cap\{r, s, t\}=\emptyset$ and $\left(\Delta w_{i} w_{j} w_{k}\right) \cap\left(\Delta w_{r} w_{s} w_{t}\right) \neq \emptyset$ where, $\triangle \mathbf{a b c}$ triangle defined by vertices $\mathbf{a}, \mathbf{b}, \mathbf{c} \in R^{D}$,

$$
\Delta a b c=\{(1-u-v) \mathbf{a}+u \mathbf{b}+v \mathbf{c} \mid 0 \leq u+v \leq 1\}
$$

Thereby, to reduce the TE, self-intersections have to be removed. He proposed the solution to detect self-intersections and redid the learning steps which caused it. His solution has disadvantages that when TE decreases, QE increases.

Obviously, trying to adjust learning algorithm to reduce both $\mathrm{QE}$ and TE is a difficult task. Thus, our solution is to re-adjust obtained map after the learning algorithm ends. In the competitive learning method $[11,25]$, the samples represented by each neuron are considered as a cluster, hence, the weight vector of the neuron will best represent for samples if it is the codebook vector of the cluster. In essence, a large QE is caused by the big difference of each data sample from its winner neuron (eq(4)), so to reduce the QE, the weight vectors must be adjusted according to the codebook vectors of the clusters and the clusters must be optimized according to the new weight vectors. This optimizing cluster method is called the competition "different elements". The "different elements" competitive process will promote weight vector of each neuron to move closer towards the weights of adjacent neighbors. This limits self-intersections status [18], so that reduces the TE.

The remaining of the paper includes: part 2 presents an overview of SOM and the quality measures of feature map; part 3 presents our solution; part 4 offers experimental results and final part is conclusions.

\section{SOM NEURAL NETWORK AND FEATURE MAP QUALITY}

\subsection{An overview of SOM}

The SOM neural network includes an input signal layer which is fully connected to an output layer called Kohonen layer (Figure.1). Kohonen layer is often organized as a two dimensional matrix of neurons. At $t$ training times, a sample $v$ is used to train the network. The training algorithm performs three steps: 


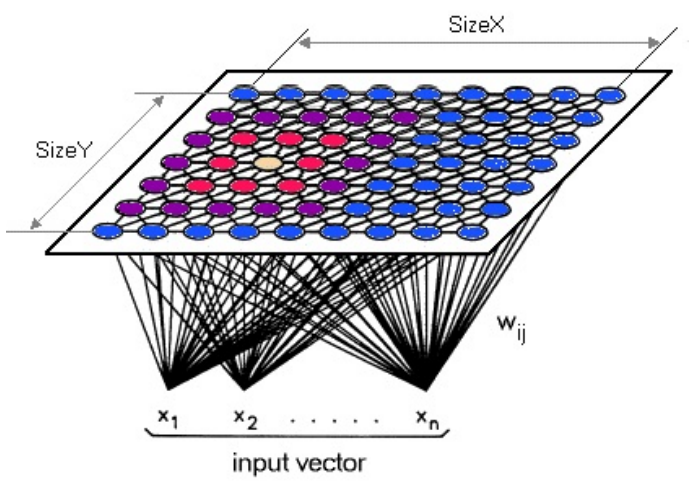

Figure 1: Illustrations of SOM.

- Step 1: Finding the best matching unit $(B M U)$ with $v$ as the eq(1).

$$
d i s t=\left\|v-\mathrm{w}_{c}\right\|=\min _{i}\left\{\left\|v-\mathrm{w}_{i}\right\|\right\}
$$

- Step 2: Calculating the neighboring radius of $B M U$ as the eq(2).

$$
N_{c}(t)=N_{0} \exp \left[-\frac{t}{\lambda}\right]
$$

where, $N_{0}$ is the initial neighboring radius;

$$
\lambda=\frac{K}{\log \left(N_{0}\right)}
$$

is the time parameter, with $K$ is the numbers of iterations.

- Step 3: Updating the weight vector of $B M U$, and neurons in the neighboring radius of $B M U$ as the eq(3).

$$
\mathrm{w}_{i}(t+1)=\mathrm{w}_{i}(t)+N_{c}(t) h_{c i}(t)\left[v-\mathrm{w}_{i}(t)\right]
$$

where,

$$
h_{c i}(t)=\exp \left[-\frac{\left\|r_{c}-r_{i}\right\|^{2}}{2 N_{c}^{2}(t)}\right]
$$

is the interpolation function over learning times, with $\left\|r_{c}-r_{i}\right\|^{2}$ is the distance from $B M U$ (neuron $c$ ) to neuron $i$ in the Kohonen layer.

\subsection{Quality measures}

Quantization Error [16]: the average difference of inputs compared to their corresponding $B M U s$.

$$
Q E=\frac{1}{T} \sum_{t=1}^{T}\left\|x(t)-\mathrm{w}_{c}(t)\right\|
$$


where, $w_{c}(t)$ is the weight vector of $B M U$ corresponding to $x(t), T$ is the total number of data samples.

Topographic Error: the numbers of the samples whose the first best matching unit $\left(B M U_{1}\right)$ and the second best matching unit $\left(B M U_{2}\right)$ are not adjacent $[15,20]$.

$$
T E=\frac{1}{T} \sum_{t=1}^{T} d(x(t))
$$

where, $d(x(t))=1$ if $B M U_{1}$ and $B M U_{2}$ of $x(t)$ are adjacent, and $d(x(t))=0$, vice verse.

Topographic Product (TP): assess the neighborhood relation preservation in the map [3]. However, TP is only reliable for linear datasets [28].

$$
T P=\sum_{i=1}^{n \times m} H_{i}
$$

where, $H_{i}=1$ if $k$ nearest neighbors of neuron $i$, which have the identical weight vector, $n \times m$ is the size of Kohonen layer.

Distortion Measure (DM): the overall quality of the SOM neural network is evaluated by energy function $E_{d}$ [17]. $E_{d}$ is used to pick out the best map from different trainings with the same dataset. However, Heskes [12] shows that $E_{d}$ can only be optimized as training set that is finite and neighboring radius fixed.

$$
E_{d}=\sum_{t=1}^{T} \sum_{i=1}^{q} h_{c i}(t)\left\|x(t)-\mathrm{w}_{i}(t)\right\|
$$

with $q$ is the number of neurons in the neighboring radius of the $B M U$ at iteration $t$.

Indeed, QE and TE are two main measures used to assess the quality of feature map [6]. The next section presents the solution to reduce the QE and TE.

\section{3. "DIFFERENT ELEMENTS" COMPETITIVE STRATEGY}

Obviously, after the training process, each neuron in the Kohonen layer will represent a data cluster including closest samples to weight vector of the neuron. So, the training dataset is divided into $s$ subsets corresponding to $s$ neurons (with $s=m \times n$, where $m \times n$ is the size of Kohonen layer). Suppose $I$ is training dataset, it yields

$$
I=\left\{I_{1}, I_{2}, \ldots, I_{s}\right\}
$$

where, $I_{i}$ is a subset including samples represented by neuron $i$ (with $i=1 . . s$ ).

Call $Q_{i}$ the difference of neuron $i$ (total of the distance of the samples of $I_{i}$ to weight vector $w_{i}$ ):

$$
Q_{i}=\sum_{v=1}^{p} d\left(x_{v}, w_{i}\right)
$$

where,

$$
d\left(x_{v}, w_{i}\right)=\left\|x_{v}-w_{i}\right\|
$$

with $x_{v} \in I_{i}, p=\left|I_{i}\right|$ is the number of samples represented by neuron $i$. 
The eq(4) is equivalent to the eq(9) below:

$$
Q E=\frac{1}{T} \sum_{i=1}^{s} Q_{i}
$$

The eq $(9)$ shows that: $\mathrm{QE}$ is minimized if $Q_{i}$ is minimized, with $\forall i=1 . . s$.

Call $c_{i}$ the codebook vector of $I_{i}\left(c_{i}\right.$ is closest to all samples of $\left.I_{i}\right)$ :

$$
c_{i}=\frac{1}{p} \sum_{v=1}^{p} x_{v}
$$

Let $Q_{i}^{c}$ the total of the distance of the samples of $I_{i}$ to the $c_{i}$.

$$
Q_{i}^{c}=\sum_{v=1}^{p} d\left(x_{v}, c_{i}\right)
$$

Hence, $Q_{i}$ is minimized if it satisfies the eq(12)

$$
Q_{i}=Q_{i}^{c} \Leftrightarrow \sum_{v=1}^{p} d\left(x_{v}, w_{i}\right)=\sum_{v=1}^{p} d\left(x_{v}, c_{i}\right)
$$

In other words, $Q_{i}$ is minimized if and only if $\mathrm{w}_{i}=c_{i}$, with $\forall i=1 . . s$

From all above, a definition about the smallest quantization error is proposed:

Definition 1. Quantization error of self-organizing map is the smallest if and only if $\mathrm{w}_{i}=c_{i}$, with $\forall i=1 . . s$, where $w_{i}$ is the weight vector of neuron $i ; c_{i}$ is the codebook vector of $I_{i}$, including samples represented by neuron $i$.

Therefore, to reduce the QE we assign $w_{i}=c_{i}$, with $i=1 . . s$. However, this leads to the consequence that some samples have to change its representative neuron, because it fits better with another neuron (compared with the neuron to which it belongs), i.e. the elements of each subset $I_{i}$ need to be redefined. The samples which need to change representative neuron are called "different elements", as the following definition:

Definition 2. $x$ is called "different elements" of neuron $i$ to neuron $j$ (with $\forall j \neq i$ ) if and only if $x \in I_{i}$ and $d\left(x, \mathrm{w}_{i}\right)>d\left(x, \mathrm{w}_{j}\right)$.

In the Figure.2, $x_{1}$ is the "different elements" of neuron $i$ to neuron $j$, with $x_{1} \in I_{i}$ : $d\left(x_{1}, \mathrm{w}_{i}\right)>d\left(x_{1}, \mathrm{w}_{j}\right) ; x_{2}$ is the "different elements" of neuron $i$ to neuron $k$, with $x_{2} \in I_{i}$ : $d\left(x_{2}, \mathrm{w}_{i}\right)>d\left(x_{2}, \mathrm{w}_{k}\right) ; x_{3} \in I_{i}$ is not the "different elements" of neuron $i$ to neuron $g$ because the condition $d\left(x_{3}, \mathrm{w}_{i}\right)>d\left(x_{3}, \mathrm{w}_{g}\right)$ is not satisfied.

From above definition results in the following theorem:

Theorem. Give $x$ is a "different elements" of neuron $i$ to neuron $j$ (with $x \in I_{i}, i \neq j$ ), we have $Q E^{*}<Q E$ if and only if $I_{i}=I_{i} \backslash x$ and $I_{j}=I_{j} \cup x$. In which $Q E$ is the quantization error before removing sample $x$ from set $I_{i}$ and updating $x$ to $I_{j}, Q E^{*}$ is the quantization error achieved after removing sample $x$ from set $I_{i}$ and updating $x$ to $I_{j}$.

Proof.

$$
\begin{aligned}
& \mathrm{Eq}(9) \Leftrightarrow Q E=\frac{1}{T}\left(Q_{1}+Q_{2}+. .+Q_{i}+. .+Q_{s}\right) \text { Let } \\
& \qquad \begin{array}{l}
Q=Q_{1}+Q_{2}+. .+Q_{i}+. .+Q_{s}=\bar{Q}+Q_{i}+Q_{j}
\end{array}
\end{aligned}
$$




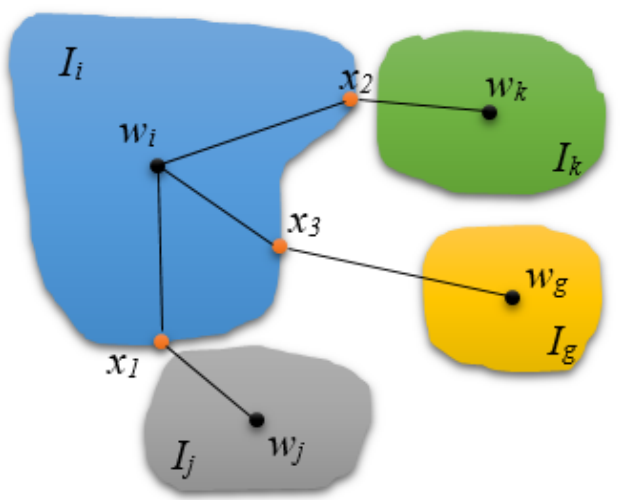

Figure 2: Illustrations of the "different elements" of neuron $i$.

with

$$
\bar{Q}=\sum_{k=1, k \neq i, k \neq j}^{s} Q_{k}
$$

Let $\bar{Q}_{i}=Q_{i}-d\left(x, \mathrm{w}_{i}\right)$ is the difference of the neuron $i$ on the set $\bar{I}_{i}=I_{i} \backslash x$

$$
\Leftrightarrow Q_{i}=\bar{Q}_{i}+d\left(x, \mathrm{w}_{i}\right)
$$

Let $\bar{Q}_{j}$ is the difference of the neuron $j$ on the set $\bar{I}_{j}=I_{j} \cup x$

$$
\bar{Q}_{j}=Q_{j}+d\left(x, \mathrm{w}_{j}\right)
$$

Calling $Q^{*}$ the total of the difference of all neurons after removing sample $x$ from set $I_{i}$ and updating $x$ to set $I_{j}$, yields:

$$
Q^{*}=\bar{Q}+\bar{Q}_{i}+\bar{Q}_{j}
$$

From the hypothesis

$$
x \in I_{i}: d\left(x, \mathrm{w}_{i}\right)>d\left(x, \mathrm{w}_{j}\right)
$$

with $i \neq j$, it yields:

$$
\bar{Q}_{i}+d\left(x, \mathrm{w}_{i}\right)>\bar{Q}_{i}+d\left(x, \mathrm{w}_{j}\right)
$$

Replacing (14) to (17), results in:

$$
\begin{gathered}
Q_{i}>\bar{Q}_{i}+d\left(x, \mathrm{w}_{j}\right) \\
\Leftrightarrow Q_{i}+Q_{j}>\bar{Q}_{i}+d\left(x, \mathrm{w}_{j}\right)+Q_{j}
\end{gathered}
$$

Replacing (15) to (18), yields:

$$
\begin{gathered}
\bar{Q}_{i}+\bar{Q}_{j}<Q_{i}+Q_{j} \\
\Leftrightarrow \bar{Q}+\bar{Q}_{i}+\bar{Q}_{j}<\bar{Q}+Q_{i}+Q_{j}
\end{gathered}
$$

Replacing (13) and (16) to (19), results in the following:

$$
Q^{*}<Q \Leftrightarrow Q E^{*}<Q E
$$


Lemma. Give $x$ is "different elements" of neuron $i\left(x \in I_{i}\right)$ to two neurons $j$ and $k$ (wih $i \neq j, i \neq k, j \neq k$ ), we have

$$
Q E_{(j)}^{*}<Q E_{(k)}^{*}
$$

if and only if $d\left(x, \mathrm{w}_{j}\right)<d\left(x, \mathrm{w}_{k}\right)$, with $Q E_{(j)}^{*}$ is the quantization error achieved if $x$ is updated to set $I_{j} ; Q E_{(k)}^{*}$ is the quantization error achieved if $x$ is updated to set $I_{k}$.

Proof.

Calling $Q_{(j)}^{*}$ the total of the difference of all neurons if $x$ is updated to $I_{j}$, yields:

$$
Q_{(j)}^{*}=Q-d\left(x, \mathrm{w}_{i}\right)+d\left(x, \mathrm{w}_{j}\right)
$$

Calling $Q_{(k)}^{*}$ the total of the difference of all neurons if $x$ is updated to $I_{k}$, results in:

$$
Q_{(k)}^{*}=Q-d\left(x, \mathrm{w}_{i}\right)+d\left(x, \mathrm{w}_{k}\right)
$$

From hypothesis

$$
d\left(x, \mathrm{w}_{j}\right)<d\left(x, \mathrm{w}_{k}\right)
$$

yields:

$$
Q_{(j)}^{*}<Q_{(k)}^{*} \Leftrightarrow Q E_{(j)}^{*}<Q E_{(k)}^{*}
$$

Thus, optimizing process of QE repeats two following steps: firstly, assign the weight vector of each neuron to the cluster's center that it represents; secondly, compete "different elements" between neurons to redetermine the data subset that each neuron represents.

The "different elements" competitive strategy is performed for all neurons aiming to optimize clusters's elements, which reduce $\mathrm{QE}$ and may also restrict the self-intersections [18] resulting in reducing TE. Figure.3 illustrates the self-intersections given by Lopez-Rubio. In particular, the weight vector of neuron $i$ matches with other units rather than adjacent neighbors $j, k, p, q$, that is the cause of self-intersections.

The "different elements" competitive process will adjust the weights of $i$ and its adjacent neighbors closer if between $i$ and its adjacent neighbors exist "different elements". Hence, if there exist "different elements" of $j, k, p, q$ to $i$, the cluster $I_{i}$ will extend towards $I_{j}, I_{k}, I_{p}, I_{q}$ and if there exist the "different elements" of $i$ to $j, k, p, q$, the clusters $I_{j}, I_{k}, I_{p}, I_{q}$ will extend towards $I_{i}$, vice verse. As a result, the corresponding cluster centers $c_{i}, c_{j}, c_{k}, c_{p}, c_{q}$ approach closer to each other. In principle, the clusters which have large differences tending to shrink (to reduce the difference), and the clusters have small differences tending to expand and move towards the clusters which have big differences.

Thus, the competition "different elements" directly reduces QE but only contributes to reduce TE. In which, QE always decreases when competition occurs, while reduction of TE depends on the number of "different elements" between the neuron which causes self-intersection and its adjacent neighbors. If the neuron which causes self-intersection is too different from its adjacent neighbors, there is non-existent of "different elements" between them, hence, the competition "different elements" can not remove the self-intersection status in this case.

We propose the IMQS algorithm which used to improve the feature map's quality, including the following steps:

- Step 1: Determine the subset $I_{i}$ of $I=\left\{I_{1}, I_{2}, . ., I_{s}\right\}$, with $i=1 . . s$ 


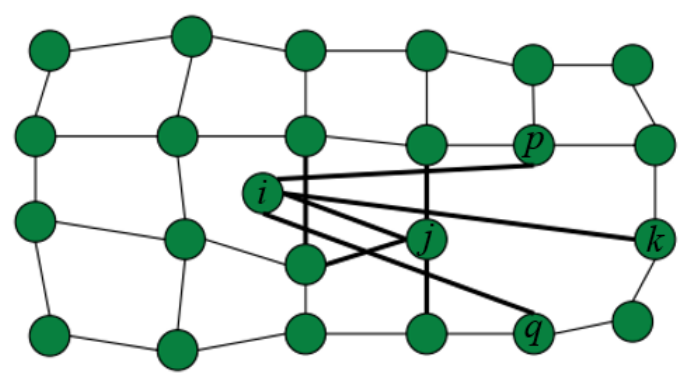

Figure 3: Illustrations of seft-intersections.

- Step 2: Repeat competition "different elements" between all neurons until satisfying one of the following stopping conditions: there is no existence of any "different elements" between all neurons or $\left\|Q E-Q E^{*}\right\|<\theta$, with $\theta$ is option threshold value.

The competition "different elements" procedure between neuron $i$ and others is as follows: With $\forall x \in I_{i}, i \neq j, i \neq k, j \neq k$,

if $d\left(x, \mathrm{w}_{i}\right)>d\left(x, \mathrm{w}_{j}\right)$ and $d\left(x, \mathrm{w}_{i}\right)>d\left(x, \mathrm{w}_{k}\right)$ and $d\left(x, \mathrm{w}_{j}\right)<d\left(x, \mathrm{w}_{k}\right)$ then

$$
\begin{gathered}
I_{i}=I_{i} \backslash x ; I_{j}=I_{j} \cup x ; \\
c_{i}=\frac{1}{\left|I_{i}\right|} \sum_{v=1}^{\left|I_{i}\right|} x_{v} ; c_{j}=\frac{1}{\left|I_{j}\right|} \sum_{v=1}^{\left|I_{j}\right|} x_{v} ; \\
\mathrm{w}_{i}=c_{i} ; \mathrm{w}_{j}=c_{j}
\end{gathered}
$$

For ease of installation, the Batch-IMQS version is recommended, allowing batch processing. Repeat two following steps until the stop condition is satisfied:

- Step 1: Determine the subset $I_{i}$ of $I=\left\{I_{1}, I_{2}, . ., I_{s}\right\}$, with $\forall i=1 . . s$.

- Step 2: Calculate the codebook vectors $c_{i}$, and assign $w_{i}=c_{i}$, with $\forall i=1 . . s$.

The Batch-IMQS is suitable for the case that the dataset's size and Kohonen layer's size are small. The next section presents experiments of the method and evaluates the effectiveness of the algorithm through measures, QE and TE.

\section{EXPERIMENTS}

The experiments are performed with two assuming datasets (XOR, Odd-Even) and eleven real datasets [1]. In each dataset, the experiment includes two phases: the first phase is training SOM neural network; the second phase is improving feature map's quality obtained of SOM by IMQS. In both phases, the QE, TE values are tracked for comparison. Kohonen layer's size is $15 \times 15$ which is used for all datasets. The IMQS algorithm has stop condition parameter $\theta=10^{-4}$, it is used to improve the map's quality obtained of SOM in two cases:

In the first case, the SOM algorithm's stop condition is when the number of training times is three times the size of the dataset (because the number of samples of some datasets is small). Table 1 is the result of QE, TE before and after applying IMQS. 


\begin{tabular}{|c|c|c|c|r|r|r|r|}
\hline \multirow{2}{*}{ No. } & \multirow{2}{*}{ Dataset } & \multirow{2}{*}{ Vectors(N) } & \multirow{2}{*}{ Features } & \multicolumn{2}{|c|}{ SOM } & \multicolumn{2}{|c|}{ IMQS } \\
\cline { 4 - 7 } & & & QE & \multicolumn{1}{c|}{ TE } & \multicolumn{1}{c|}{ QE } & \multicolumn{1}{c|}{ TE } \\
\hline 1 & XOR & 4125 & 2 & 0.0483 & 0.9707 & 0.0311 & 0.9350 \\
\hline 2 & Odd-Even & 400 & 8 & 130.3291 & 0.85 & 67.6296 & 0.39 \\
\hline 3 & Iris & 150 & 4 & 0.5593 & 0.8 & 0.2098 & 0.4467 \\
\hline 4 & Glass & 214 & 9 & 4.0375 & 0.3879 & 1.6695 & 0.3411 \\
\hline 5 & Flame & 240 & 2 & 0.9542 & 0.5708 & 0.3723 & 0.4125 \\
\hline 6 & Pathbased & 300 & 2 & 3.2396 & 0.5833 & 0.9751 & 0.5067 \\
\hline 7 & Spiral & 312 & 2 & 3.0021 & 0.5769 & 0.8925 & 0.4071 \\
\hline 8 & Jain & 373 & 2 & 1.6923 & 0.4987 & 0.7313 & 0.4745 \\
\hline 9 & Compound & 399 & 2 & 1.6329 & 0.6090 & 0.7192 & 0.6040 \\
\hline 10 & Aggregation & 788 & 2 & 2.3739 & 0.5584 & 1.0257 & 0.4251 \\
\hline 11 & R15 & 600 & 2 & 0.6842 & 0.8917 & 0.2532 & 0.7283 \\
\hline 12 & D31 & 3100 & 2 & 4.8514 & 0.6468 & 1.0631 & 0.4958 \\
\hline 13 & Wine & 178 & 13 & 63.4801 & 0.3146 & 13.0856 & 0.2753 \\
\hline
\end{tabular}

Table 1: Results of QE, TE with training times of SOM by $3 \times N$.

\begin{tabular}{|c|c|c|c|r|r|r|r|}
\hline \multirow{2}{*}{ No. } & \multirow{2}{*}{ Dataset } & \multirow{2}{*}{ Vectors(N) } & \multirow{2}{*}{ Param k } & \multicolumn{2}{|c|}{ SOM } & \multicolumn{2}{c|}{ IMQS } \\
\cline { 4 - 7 } & & & QE & \multicolumn{1}{c|}{ TE } & \multicolumn{1}{c|}{ QE } & \multicolumn{1}{c|}{ TE } \\
\hline 1 & XOR & 4125 & 10 & 0.0385 & 0.9685 & 0.0294 & 0.9372 \\
\hline 2 & Odd-Even & 400 & 60 & 98.5358 & 0.8975 & 56.5497 & 0.4925 \\
\hline 3 & Iris & 150 & 30 & 0.3553 & 0.8733 & 0.1177 & 0.6733 \\
\hline 4 & Glass & 214 & 60 & 2.9664 & $\mathbf{0 . 4 4 3 9}$ & 1.4712 & $\mathbf{0 . 4 6 2 6}$ \\
\hline 5 & Flame & 240 & 30 & 0.4684 & 0.9292 & 0.2184 & 0.8625 \\
\hline 6 & Pathbased & 300 & 30 & 0.9543 & 0.8367 & 0.4825 & 0.74 \\
\hline 7 & Spiral & 312 & 30 & 1.1807 & 0.8462 & 0.5970 & 0.7981 \\
\hline 8 & Jain & 373 & 30 & 0.7374 & 0.8794 & 0.4187 & 0.8231 \\
\hline 9 & Compound & 399 & 30 & 0.8649 & 0.9599 & 0.5373 & 0.8747 \\
\hline 10 & Aggregation & 788 & 30 & 1.1502 & 0.8363 & 0.7541 & 0.75 \\
\hline 11 & R15 & 600 & 30 & 0.2724 & 0.8817 & 0.1623 & 0.76 \\
\hline 12 & D31 & 3100 & 10 & 1.7487 & 0.7916 & 0.7336 & 0.7103 \\
\hline 13 & Wine & 178 & 60 & 19.5587 & 0.4270 & 6.2342 & 0.3764 \\
\hline
\end{tabular}

Table 2: Results of QE, TE with training times of SOM by $k \times N$.

Table 1 shows that all the QE and TE values of algorithm IMQS decrease compared with the corresponding values of SOM. The average reduction ratio of $\mathrm{QE}$ is $61.2 \%$ and $\mathrm{TE}$ is $20.6 \%$.

In the second case, the number of training times of the SOM algorithm is increased to test the ability of its convergence compared with IMQS. Although the number of training times of datasets increases largely ( $k$ times the size of the dataset), the QE and TE values of SOM in Table 2 are still greater than the QE, TE values of IMQS in Table 1. This means that, instead of increasing the number of SOM's training times too many, the IMQS algorithm should be used to optimize the feature map to achieve more optimal $\mathrm{QE}$ and $\mathrm{TE}$ values, in less time. In fact, the larger number of SOM training times is, the less fluctuation of QE and TE will be, however, only QE tends to decrease 


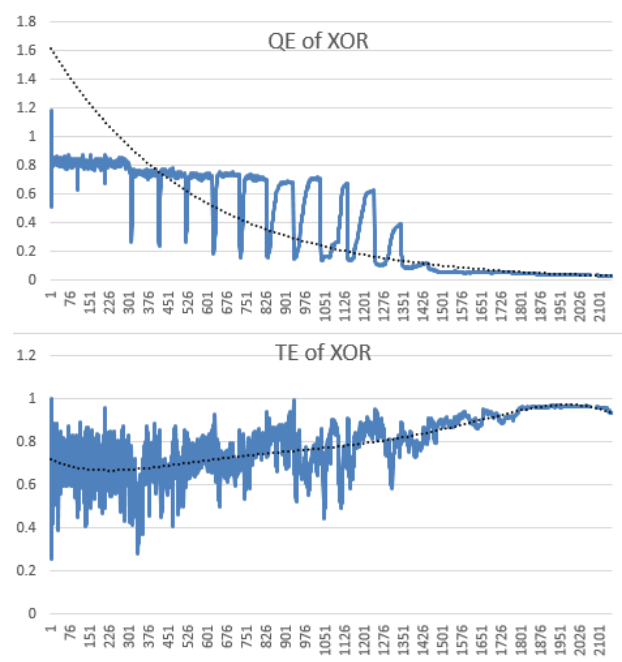

Figure 4: Chart of QE and TE of XOR.

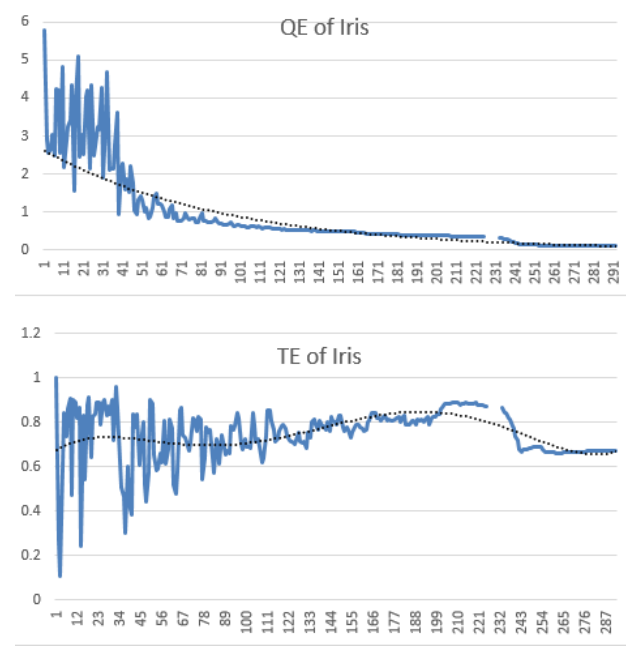

Figure 6: Chart of QE and TE of Iris.

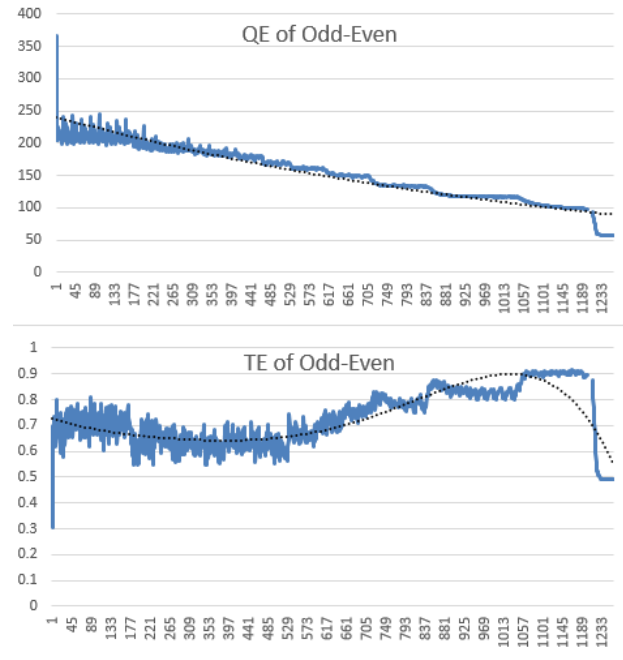

Figure 5: Chart of QE and TE of Odd-Even.

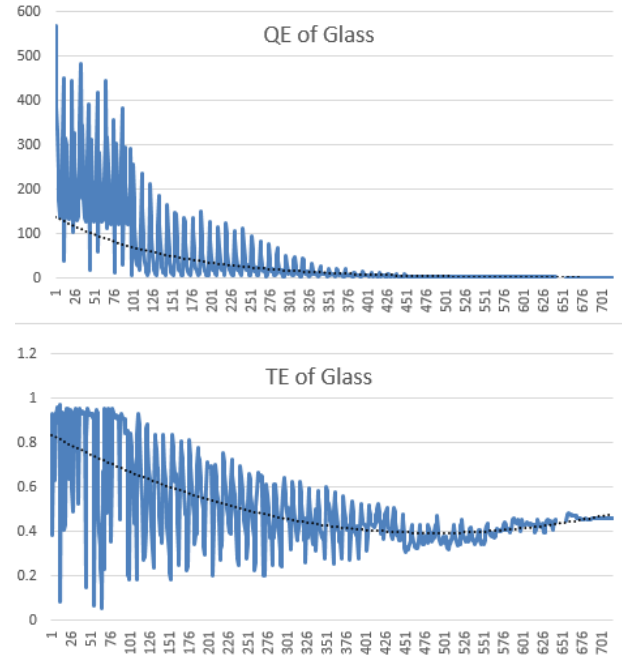

Figure 7: Chart of QE and TE of Glass.

gradually, TE tends to increase gradually. Thus, further increasing the number of training SOM is not as effective as using IMQS.

In the Table 2, the reduction ratio of QE, TE (of IMQS compared with SOM) is less than that in Table 1. In which, the average reduction ratio of $\mathrm{QE}$ is $47.5 \%, \mathrm{TE}$ is $11.8 \%$. It is caused by when the training times of SOM increases, the data representation accuracy increases (in other words, the difference reduces or the number of "different elements" reduces), but the nature of IMQS algorithm is trying to decrease the difference to reduce QE and TE, so IMQS will be less effective if the number of "different elements" is small. In the result of Glass dataset, the IMQS algorithm couldn't reduce TE while QE still decreased. This shows that the self-intersections are not removed (the cause is explained above). The reason that TE of IMQS rises slightly compared to TE of SOM is the correlation between $\mathrm{QE}$ and TE [24]. 


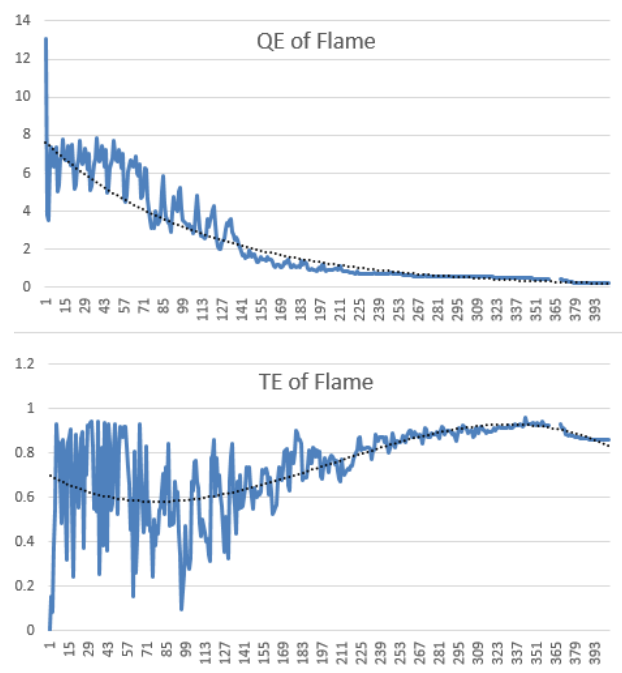

Figure 8: Chart of QE and TE of Flame.

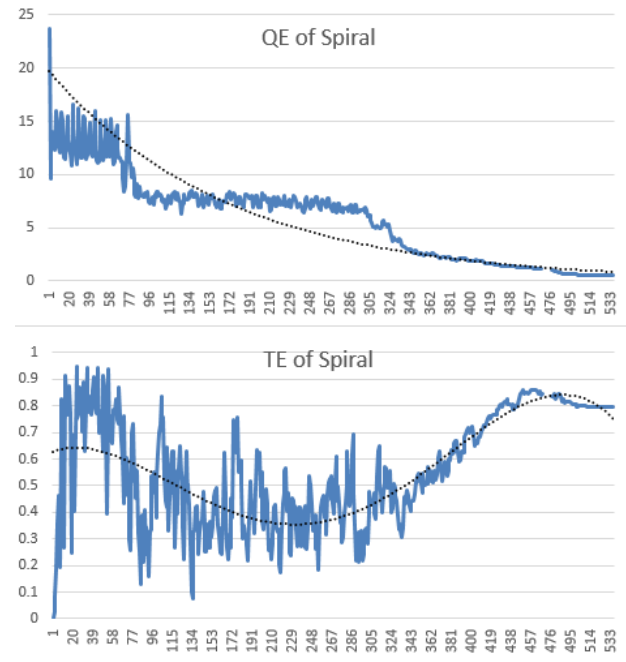

Figure 10: Chart of QE and TE of Spiral.

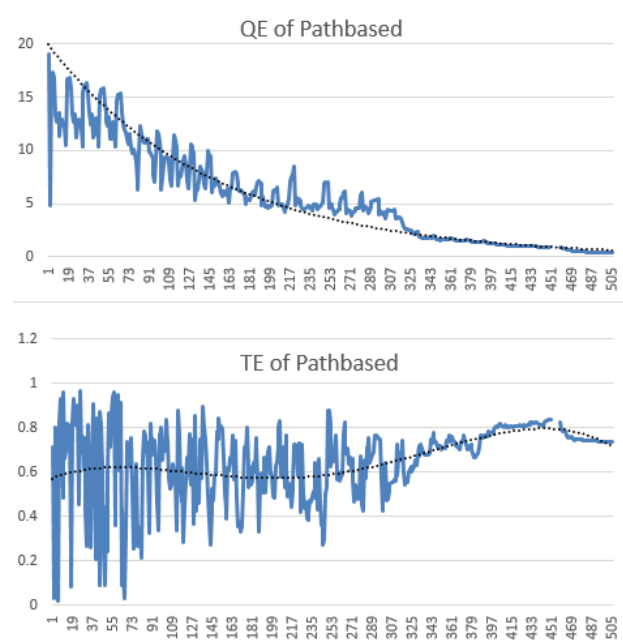

Figure 9: Chart of QE and TE of Pathbased.

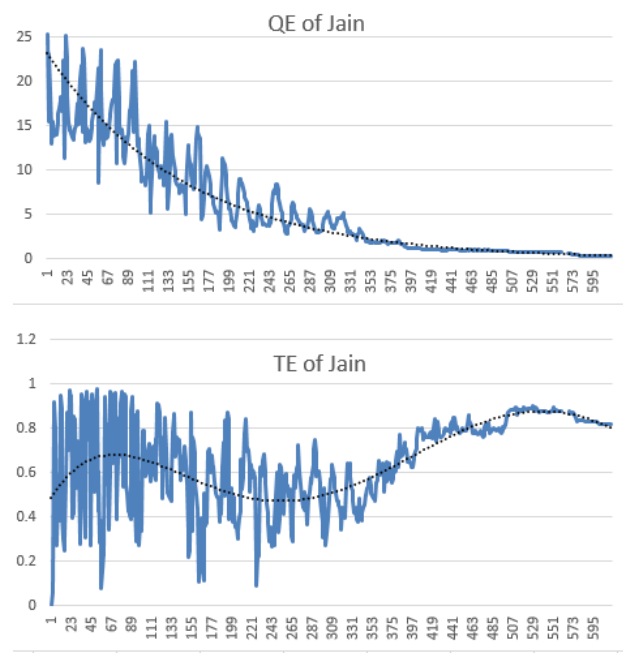

Figure 11: Chart of QE and TE of Jain.

The figures from Figure.4 to Figure.16 are the charts which present the fluctuation process of $\mathrm{QE}$ and TE of datasets in the second test case (details of all experimental results are presented in appendix). In particular, the vertical axis represents the value of the measures; a horizontal axis represents the times of training; the gap in the line represents the ending of SOM and the beginning of IMQS algorithm; dashed line shows trends.

Observing the charts of SOM (from the starting point to the gap) it is found that: Initially, the values of $\mathrm{QE}$ and TE fluctuates dramatically, but when the number of training times increases, the fluctuation decreases. It is because the neighboring radius gradually decreases in the training process. There is a tradeoff between QE and TE, where QE decreases gradually to 0, whereas, TE increases gradually to 1 .

The charts of IMQS (from the gap to the end) is much shorter than the chart of SOM, because the 


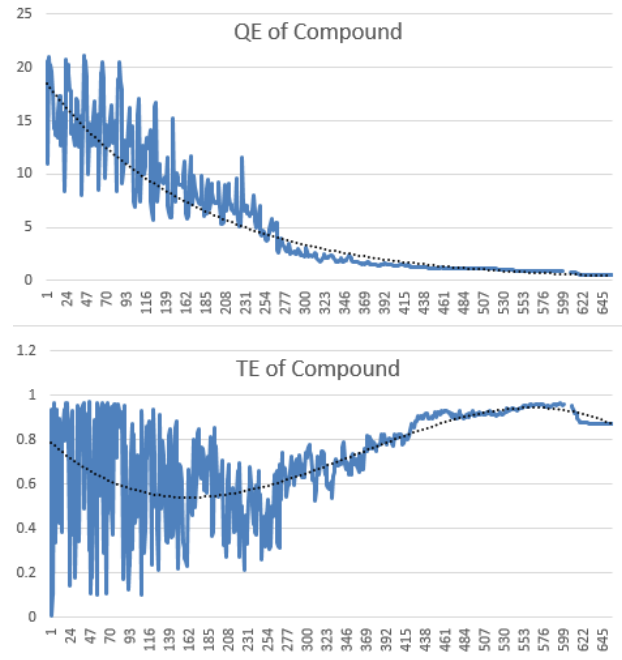

Figure 12: Chart of QE and TE of Compound.

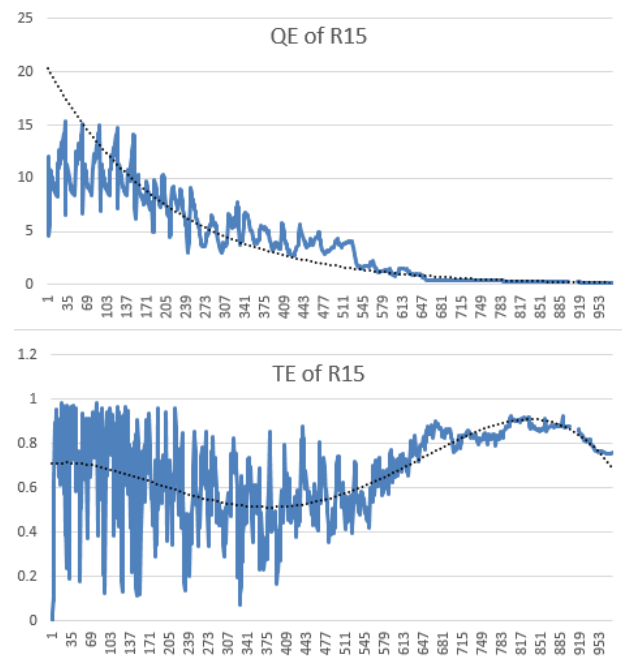

Figure 14: Chart of QE and TE of R15.

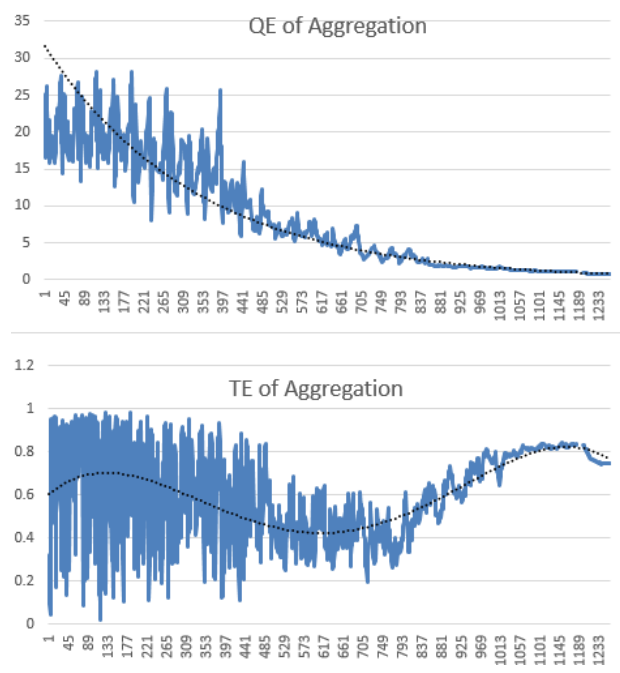

Figure 13: Chart of QE and TE of Aggregation.

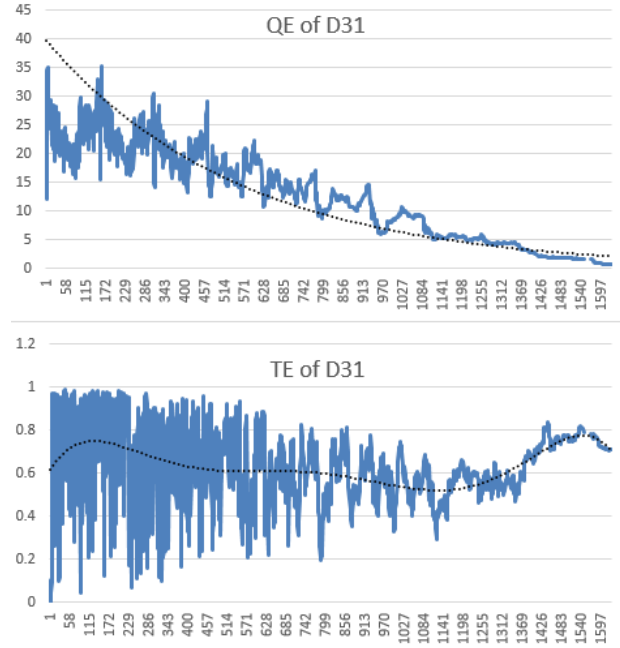

Figure 15: Chart of QE and TE of D31.

IMQS algorithm converges quite quickly. QE steadily decreases with all datasets, but TE has different reduction rate with each dataset because it depends on the ability to remove the self-intersections status. In most of the datasets, IMQS's TE has a downward trend. It is rare that TE does not reduce.

In both cases, the reduction ratio of $\mathrm{TE}$ is always less than the reduction ratio of $\mathrm{QE}$ because $\mathrm{QE}$ always decreases when competing "different elements" between all neurons occurring, but TE may only reduces when competing "different elements" between the neurons which cause self-intersection and its adjacent neighbors occurs.

Beside experiments on 13 datasets above, some experiments on other datasets such as image data, sound and the results are similar are also conducted. 


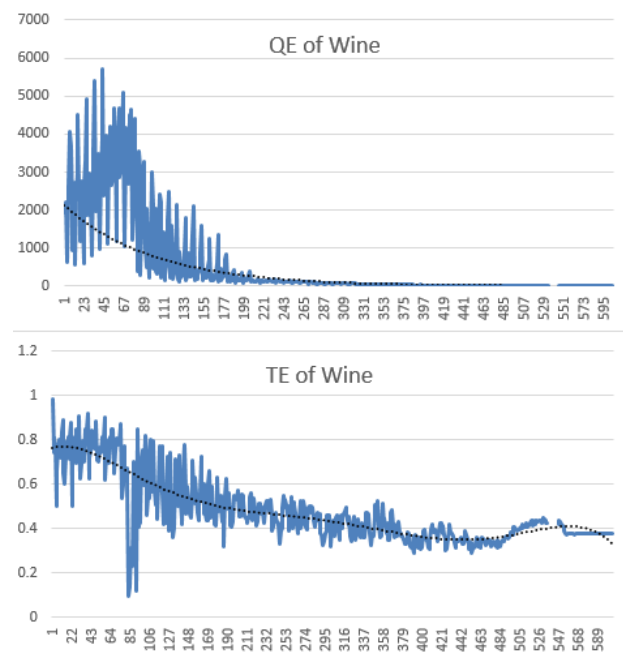

Figure 16: Chart of QE and TE of Wine.

\section{CONCLUSIONS}

Theoretically, the original SOM and some improved SOMs can achieve good map quality if the suitable configuration parameters of the network are determined. However, in reality, it is time consuming and very difficult to perform because we must be "trying error" several times to determine the parameter's value of each dataset. Thus, the map obtained by the unsuitable parameters is a common outcome in practice. Therefore, our solution does not aim to adjust the configuration parameters as well as learning algorithm, but to optimize obtained map after learning algorithm ends. The final obtained map has better quality because QE plummets and TE decreases in most cases, except that the number of "different elements" between the neuron which cause self-intersection and its adjacent neighbors is small or non-existed. Although our method can not reduce TE in all cases, but in reality, this is an effective method to improve the feature map quality. Compared with the method by Kamimura [14], our method has a larger reduction rate of $\mathrm{QE}$ and $\mathrm{TE}$ and can be applied to all datasets without valuating private parameters for each dataset. In addition, the proposed method completely appropriates the original SOM or any other variants of SOM, because we do not impact on learning algorithm but only optimize the obtained maps of those models.

The reduction ratio of TE may greater if IMQS algorithm prioritizes to competition between adjacent neighbors first, then to the remaining neurons (Batch-IMQS is unable to prioritize competing since the data is processed in batch), because self-intersection status will be eliminated sooner when the weights of the adjacent units move closer together faster. In fact, the reduction of TE is only effective if we exclude self-intersection status as soon as it appears in the training process.

\section{APPENDIX}

The following link provides detail of the experiments, including the value of $\mathrm{QE}$, TE tracked in real time and the charts which show the process of the fluctuation of $\mathrm{QE}$ and TE in both experimental cases.

http://www.mediafire.com/view/hrdpyoc3znot5y7/experimental_results.xlsx 


\section{ACKNOWLEDGMENT}

The author would like to thank the scientists of the Information Technology Institute - Vietnam Academy of Science and Technology for helping him during the time he had been studying there.

\section{REFERENCES}

[1] [Online]. Available: http://cs.joensuu.fi/sipu/datasets/

[2] H. Bauer, M. Herrmann, and T. Villmann, "Neural maps and topographic vector quantization," Neural Netw, vol. 12, no. 4-5, pp. 659-676, 1999.

[3] H. Bauer and K. Pawelzik, "Quantifying the neighborhood preservation of self organizing feature maps," IEEE Transactions on Neural Networks, vol. 3, no. 4, pp. 570-579, Jul 1992.

[4] E. Berglund and J. Sitte, "The parameterless self-organizing map algorithm," Neural Netw IEEE Trans, vol. 17, no. 2, pp. 305-316, 2006.

[5] A. César Astudillo and B. John Oommen, "Topology-oriented self-organizing maps: A survey," Pattern Analysis and Applications, vol. 17, no. 2, pp. 223-248, May 2014.

[6] M. Chattopadhyay, P. K. Dan, and S. Mazumdar, "Application of visual clustering properties of self organizing map in machine-part cell formation," Applied Soft Computing, vol. 12, no. 2, pp. 600-610, 2012.

[7] J. A. Flanagan, "Self-organization in kohonen's som," Neural Networks, vol. 9, no. 7, pp. 11851197, 1996.

[8] E. Germen, "Increasing the topological quality of kohonen's self organizing map by using a hit term," in Neural Information Processing, Proceedings of the 9th International Conference on (ICONIP '02), vol. 2. Singapore: IEEE, Nov 18-22 2002, pp. 930-934.

[9] —_ "Improving the resultant quality of kohonens self organizing map using stiffness factor," in Advances in Natural Computation, Lecture Notes in Computer Science (First International Conference, ICNC 2005), vol. 3610. Changsha, China: Springer Berlin Heidelberg, August 27-29 2005, pp. 353-357.

[10] E. Germen and S. Bilgen, "A statistical approach to determine the neighborhood function and learning rate in self-organizing maps," in Proc. ICONIP97. Springer, 1997, pp. 334-337.

[11] S. Grossberg, "Competitive learning: From interactive activation to adaptive resonance," Elsevier, vol. 11, no. 1, pp. 23-63, JanuaryMarch 1987.

[12] T. Heskes, "Energy functions for self-organizing maps," in Kohonen Maps, S. K. E. Oja, Ed. Amsterdam: Elsevier Science, 1999, pp. 303-316.

[13] C. Kahraman, Computational Intelligence Systems in Industrial Engineering, 1st ed., C. E. Kahraman, Ed. Atlantis Press, 2012, vol. 6.

[14] R. Kamimura, "Input information maximization for improving self-organizing maps," Applied Intelligence, vol. 41, no. 2, pp. 421-438, Mar 202014.

[15] K. Kiviluoto, "Topology preservation in self-organizing maps," in Neural Networks, IEEE International Conference on (ICNN96), vol. 1. Washington, DC: IEEE, Jun 3-6 1996, pp. 294-299. 
[16] T. Kohonen, Self-Organizing Maps, 3rd ed. Springer-Verlag, 2001.

[17] J. Lampinen and E. Oja, "Clustering properties of hierarchical self-organizing maps," Journal of Mathematical Imaging and Vision, vol. 2, no. 2-3, pp. 261-272, November 1992.

[18] E. Lopez-Rubio, "Improving the quality of self-organizing maps by self-intersection avoidance," Neural Networks and Learning Systems, IEEE Transactions on, vol. 24, no. 8, pp. 1253-1265, Aug 2013.

[19] F. Mulier and V. Cherkassky, "Statistical analyses of self-organization," Neural Networks, vol. 8, no. 5, pp. 717-727, 1995.

[20] J. I. Mwasiagi, H. XiuBao, W. XinHou, and C. Qing-dong, "The use of k-means and kohonen self organizing maps to classify cotton bales," in Beltwide Cotton Conferences (BWCC'07), New Orleans, Louisiana, January 9-12 2007.

[21] A. Neme, E. Chavez, A. Cervera, and V. Mireles, "Decreasing neighborhood revisited in selforganizing map," in Artificial Neural Networks - ICANN 2008, vol. 5163. Prague, Czech Republic: Springer Berlin Heidelberg, September 3-6 2008, pp. 671-679.

[22] A. Neme and P. Miramontes, "Self-organizing map formation with a selectively refractory neighborhood," Neural Processing Letters, vol. 39, no. 1, pp. 1-24, February 2014.

[23] D. Polani, "Measures for the organization of self-organizing maps," Studies in Fuzziness and Soft Computing, vol. 78, pp. 13-44, 2002.

[24] G. Pölzlbauer, "Survey and comparison of quality measures for self-organizing maps," in Proceedings of the Fifth Workshop on Data Analysis (WDA - 04), J. Paralič, G. Pölzlbauer, and A. Rauber, Eds. Sliezsky dom, Vysoké Tatry, Slovakia: Elfa Academic Press, June 24-27 2004, pp. $67-82$.

[25] D. E. Rumelhart and D. Zipser, Feature Discovery By Competitive Learning. MA, USA: MIT Press Cambridge, 1986, vol. 1, ch. 5, pp. 151-193.

[26] Y. Sun, "On quantization error of self-organizing map network," Neurocomputing, vol. 34, no. 1-4, pp. 169-193, September 2000.

[27] A. E. Uriarte and D. F. Martín, "Topology preservation in som," Int J Appl Math Comput Sci, vol. 1, no. 1, pp. 19-22, 2005.

[28] T. Villmann, R. Der, and T. Martinez, "A new quantitative measure of topology preservation in kohonen's feature maps," in Proceedings of the IEEE International Conference on Neural Networks 94, vol. 2. Orlando, Florida, USA: IEE, 1994, pp. 645-648.

[29] S. Wang and H. Wang, "Knowledge discovery through self-organizing maps: Data visualization and query processing," Knowledge and Information Systems, vol. 4, no. 1, pp. 31-45, 2002.

Received on June 17 - 2015

Revised on September 14 - 2015 